Pacific Journal of Mathematics

REPRESENTING CODIMENSION-ONE HOMOLOGY CLASSES . 


\title{
REPRESENTING CODIMENSION-ONE HOMOLOGY CLASSES BY EMBEDDED SUBMANIFOLDS
}

\author{
Wullam H. MeEks III AND Julie Patrusky
}

In this paper we prove a general theorem on representing codimension-one homology classes on compact manifolds. Our theorem gives an elementary proof of the classical result that a one-dimensional homology class on a compact orientable surface can be represented by an embedded circle precisely when the class is primitive. We will call a homology class primitive if it is the zero class or if it is not a nontrivial multiple of another class.

Our representation theorem is motivated by our earlier work [2] on the classical situation. For compact orientable surfaces we developed a simple algorithm for representing primitive homology classes by embedded circles. This algorithm is also useful for proving other results related to two and three dimensional topology which are not implied by the general result here. Recently Mark Meyerson [3] has given another proof of the two dimensional case by applying Lickorish "twist" homeomorphisms to a fixed homology class.

The following theorem deals with the decomposition of a $n$ dimensional manifold $M^{n}$ by an embedded submanifold $N^{n-1}$ which represents a homology class $\delta \in H_{n-1}(M, Z)$. We will say that such a representation $N$ is minimal if there is no other representation $N^{\prime}$ of $\delta$ having fewer path components.

Representation Theorem. Suppose $M$ is a compact orientable piecewise linear $n$-dimensional manifold and $\gamma \in H_{n-1}(M, Z)$ is a primitive nonzero class.

1. The class $\delta=\kappa \gamma$ has a minimal representation by a submanifold.

2. If $N$ is a minimal representation for $\delta=\kappa \gamma$ then each path component of $M-N$ has two ends and the number of path components of $N=|N|$ is $\kappa$.

Proof. The Poincaré dual to $\delta=\kappa \gamma$ can be represented by a piecewise linear mapping $P(\delta): M \rightarrow S^{1}$. The standard duality theorems imply that wherever $r \in S^{1}$ is a regular value for $P(\delta)$, then $P^{-1}(r) \subset M$ is an embedded submanifold representing $\delta$. Hence any $\delta \in H_{n-1}(M, Z)$ has a minimal representative.

Suppose now that $N$ is an oriented path-connected submanifold of $M$ with $[N] \neq 0 \in H_{n-1}(M, Z)$. Since $N$ is path connected and nontrivial 
on homology then $M-N$ is path connected. Hence there is an embedded circle $\sigma \subset M$ with $[\sigma] \cap[N]=+1$, where $\cap$ is the intersection pairing on homology. This implies that $[N]$ is primitive.

Definition. If $N \subset M$ is an embedded submanifold, and $U$ is a path component of $M-N$, then $T=e n d$ closure of $U$ is formed by placing a compact boundary on each end of $U$.

Let $N$ be an embedded submanifold representing $\delta \in$ $H_{n-1}(M, Z)$. Suppose $T$ is the end closure of an oriented path component of $M-N$ with at least three ends, $E_{1}, E_{2}, E_{3}$, coming from cuts along distinct path components $N_{1}, N_{2}, N_{3}$ of $N$. Orient the end $E_{i}$ as the respective component $N_{1}$ is oriented. Let $\gamma_{1}$ be a path joining $p_{1} \in E_{1}$ to $p_{2} \in E_{2}$, and let $\gamma_{2}$ be a path joining $p_{2} \in E_{2}$ to $p_{3} \in E_{3}$ with $\gamma_{1} \cap \gamma_{2}=\left\{p_{2}\right\}$. If $\operatorname{sgn}\left(\gamma_{1} \cap E_{1}\right) \neq \operatorname{sgn}\left(\gamma_{1} \cap E_{2}\right)$, then we can take the connected sum of $N_{1}$ and $N_{2}$ in $M$ along $\gamma_{1}$, and similarly, if $\operatorname{sgn}\left(\gamma_{2} \cap\right.$ $\left.E_{2}\right) \neq \operatorname{sgn}\left(\gamma_{2} \cap E_{3}\right)$, we can connect $N_{2}$ and $N_{3}$ in $M$ along $\gamma_{2}$. If neither of these cases hold, form the composite path $\gamma_{3}=\gamma_{1}^{\circ} \gamma_{2}^{-1}$. Clearly, $\operatorname{sgn}\left(\gamma_{3} \cap E_{1}\right) \neq \operatorname{sgn}\left(\gamma_{3} \cap E_{3}\right)$. Since the normal bundle to $E_{2}$ is trivial, we may push $\gamma_{3}$ off of $E_{2}$ and take the connected sum of $N_{1}$ and $N_{3}$ in $M$ along this variation of $\gamma_{3}$.

A slight change in the above argument shows that the condition that $E_{1}, E_{2}, E_{3}$ come from cuts along distinct path components of $N$ is not needed. Hence, if $N$ is a minimal representative of $\delta$, then each path component of $M-N$ has two ends. This implies that $[N]=|N| \cdot\left[N_{1}\right]$, where $N_{1}$ is a path component of $N$. Since $N_{1}$ is primitive, the theorem is proved.

\section{REFERENCES}

1. D. R. J. Chillingworth, Winding numbers on surfaces, II., Math. Ann., 199 (1972), 142-153.

2. W. Meeks and J. Patrusky, Representing homology classes by embedded circles on a compact surface. To be published in Illinois J. Math.

3. M. D. Meyerson, Representing homology classes of closed orientable surfaces. To be published in Proc. A.M.S.

4. B. L. Reinhart, Algorithms for Jordan curves on compact surfaces, Ann. Math., 75 (1962), 209-222.

Received December 12, 1974, and in revised form July 23, 1976. This work was supported under NSF Grant No. MCS 76-07147.

University of CAlifornia, Los Angeles

AND

UNIVERSITY OF CALIFORNIA, BERKELEY 



\section{Pacific Journal of Mathematics}

Vol. 68, No. 1

March, 1977

Richard Julian Bagby, On $L^{p}, L^{q}$ multipliers of Fourier transforms . .......

Robert Beauwens and Jean-Jacques Van Binnebeek, Convergence theorems in

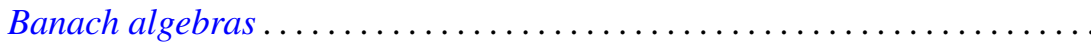

James Cyril Becker, Skew linear vector fields on spheres in the stable

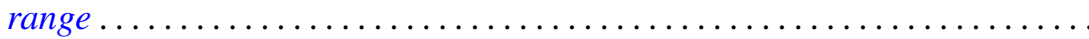

Michael James Beeson, Continuity and comprehension in intuitionistic formal

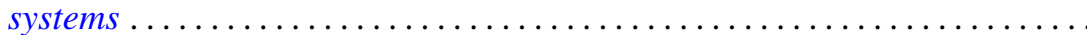

James K. Deveney, Generalized primitive elements for transcendental field

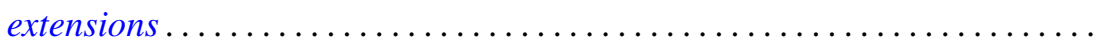

Samuel S. Feder, Samuel Carlos Gitler and K. Y. Lam, Composition properties

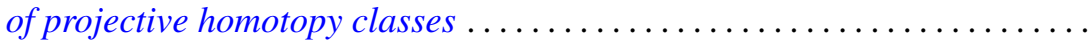

Nathan Jacob Fine, Tensor products of function rings under composition ......

Benno Fuchssteiner, Iterations and fixpoints . . . . . . . . . . . . . .

Wolfgang H. Heil, On punctured balls in manifolds

Shigeru Itoh, A random fixed point theorem for a multivalued contraction

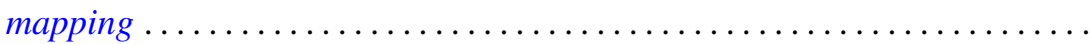

Nicolas P. Jewell, Continuity of module and higher derivations . . . . . . ......

Roger Dale Konyndyk, Residually central wreath products . . . . . . . . . . .

Linda M. Lesniak and John A. Roberts, On Ramsey theory and graphical

parameters.

Vo Thanh Liem, Some cellular subsets of the spheres.

Dieter Lutz, A perturbation theorem for spectral operators

P. H. Maserick, Moments of measures on convex bodies ... . . .

Stephen Joseph McAdam, Unmixed 2-dimensional local domains . .

D. B. McAlister and Norman R. Reilly, E-unitary covers for inverse semigroups...

William H. Meeks, III and Julie Patrusky, Representing codimension-one

homology classes by embedded submanifolds . . .

Premalata Mohapatro, Generalised quasi-Nörlund summability . .

Takahiko Nakazi, Superalgebras of weak-*Dirichlet algebras .

Catherine Louise Olsen, Norms of compact perturbations of operators .

William Henry Ruckle, Absolutely divergent series and isomorphism of

subspaces. II.

Bernard Russo, On the Hausdorff-Young theorem for integral operators .

Arthur Argyle Sagle and J. R. Schumi, Anti-commutative algebras and

homogeneous spaces with multiplications ............

Robert Evert Stong, Stiefel-Whitney classes of manifolds .

D. Suryanarayana, On a theorem of Apostol concerning Möbius functions of

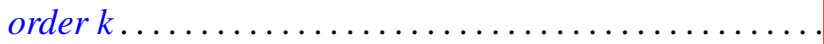

Yoshio Tanaka, On closedness of $C$ - and $C^{*}$-embeddings . . 